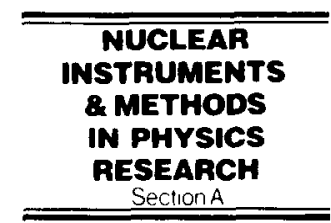

\title{
A Cherenkov free electron laser with high peak power
}

\author{
P.J.M. van der Slot ${ }^{\mathrm{a},}{ }^{*}$, J. Couperus ${ }^{\text {a }}$, W.J. Witteman ${ }^{\mathrm{b}}$, A.N. Lebedev ${ }^{\mathrm{c}}$, E.G. Krastelev ${ }^{\mathrm{c}}$, \\ A.V. Agafonov ${ }^{\mathrm{c}}$, V.S. Voronin ${ }^{\mathrm{c}}$, V.A. Krasnopolsky ${ }^{\mathrm{d}}$ \\ ${ }^{a}$ Nederlands Centrum voor Laser Research, P.O. Box 2662, 7500 CR, Enschede, The Netherlands \\ ${ }^{\mathrm{b}}$ Department of Applied Physics, Unicersity of Twente, P.O. Box 217, 7500 AE, Enschede, The Netherlands \\ ${ }^{\mathfrak{c}}$ P.N. Lebedev Physical Institute, Leninsky Prosp. 53, Moscow 117924, Russian Federation \\ ${ }^{`}$ Moscow Radiotechnical Institute, Varshat'skoe Sh. 132, Moscow 113519, Russian Federation
}

\begin{abstract}
A Cherenkov FEL can be a suitable source for radiation from the millimeter wavelength region down to the far infrared. With only a few different dielectric materials the laser can range from $6 \mathrm{~mm}$ down to $\approx 600 \mu \mathrm{m}$. Nonlinear theory shows, for an amplifier configuration, power levels of about $100 \mathrm{~kW}$ in the far infrared up to several MW at millimeter wavelengths. Good coupling with the evanescent wave supported by the dielectrically lined waveguide requires a thin walled annular beam of good quality. The characteristics of such an electron gun, a special design for a Cherenkov FEL with parameters given in this paper, will be presented.
\end{abstract}

\section{Introduction}

Different types of sources are available in the far infrared and millimeter wavelength region [1]. They all have the disadvantage that they are only tunable over a relative small region or have a low to very low power. To cover the complete wavelength region many different devices are needed. The Free Electron Laser (FEL) could provide an alternative. Of the different types of FELs, the Cherenkov FEL (and related devices) [2] seem suitable for producing radiation in the far infrared and millimeter wavelength region. Some characteristics of such a device will be presented here. A Chcrenkov FEL is a rcasonably compact device due to the fact that the generated wavelength decreases with decreasing electron beam energy. However, it requires a good quality thin annular electron beam. A special thermionic electron gun was designed and constructed to produce such an electron beam. This gun will be presented in the last section. All parameters given in this paper reflect design parameters of the laser under construction. Much of the pioneering work on Cherenkov FELs has been done by Walsh and his coworkers (see, e.g. Refs. [3,4]).

\footnotetext{
${ }^{*}$ Corresponding author. Tel +3153893965 , fax +3153338 065, e-mail peter@qe.tn.utwente.nl.
}

\section{Basic properties}

Consider a dielectrically lined waveguide with inner radius $r_{\mathrm{d}}$ of a dielectric medium with thickness $d$, the waveguide radius being $r_{\mathrm{d}}+d$. Two types of waves are supported by this waveguide. A more or less normal waveguide mode whenever the phase velocity of the wave is above and an evanescent wave in the vacuum region for waves with a phase velocity below the speed of light in vacuum. This can be seen in Fig. 1 where the dispersion relation [4] is plotted for a dielectrically lined waveguide for the three lowest modes, together with the radial profile of the axial electric field component of the $\mathrm{TM}_{01}$ mode at two different frequencies corresponding to the two different types of waves. It should be noted at this point that dividing the waveguide modes into two classes (TE and TM) is only possible for axisymmetric modes (which we are considering here). The parameters are $\epsilon_{\mathrm{r}}=2.1, r_{\mathrm{d}}=$ $3.25 \mathrm{~mm}$ and $d=0.25 \mathrm{~mm}$. Also shown as dotted lines in Fig. 1 are the two asymptotes corresponding to $\omega /(c k)=1$ and $\omega /(c k)=\epsilon_{\mathrm{I}}^{-1 / 2}$, i.e. the light lines in vacuum and a dielectric medium and the beam line $(\omega=c \beta k)$. Several properties can be concluded from this figure. First of all, interaction between the electron beam and a wave is only possible if the beam energy $\gamma$ is above a threshold value $\gamma_{\mathrm{T}}^{2}=\epsilon_{\mathrm{r}} /\left(\epsilon_{\mathrm{r}}-1\right)$. As the scale of decay for the evanescent waves is the radiation wavelength, good coupling can only be maintained for a beam moving closely along the dielectric medium. A hollow beam would have a larger growth rate compared to a solid beam due to a better overlap with 


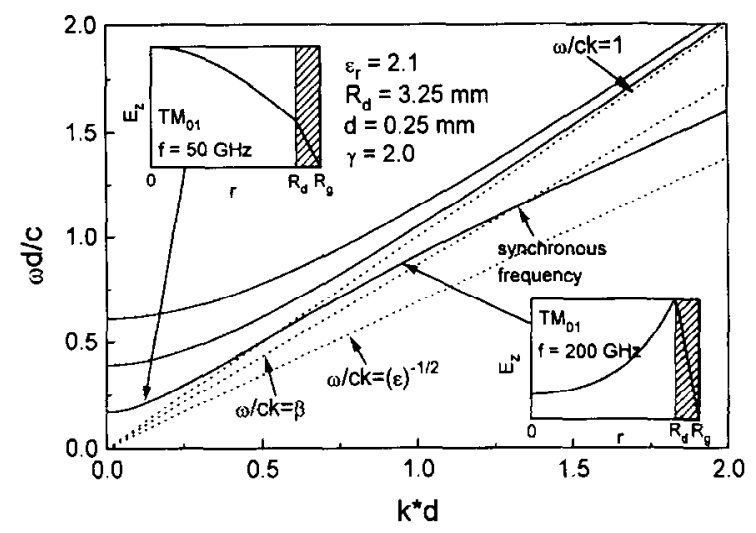

Fig. 1. Solution of the dispersion relation. The inserts show the radial dependence of the longitudinal component of the electric field for a $\mathrm{TM}_{01}$ mode at two different frequencies.

the radiation field. Finally, the synchronous frequency (see Fig. 1) increases with decreasing beam energy.

This synchronous frequency has been calculated [4] as a function of the beam accelerating voltage. These calculations show that for $\epsilon_{\mathrm{r}}=2.1, d=0.25 \mathrm{~mm}$ and a fixed geometry the laser can be tuned from $\approx 220 \mathrm{GHz}$ to $\approx 450 \mathrm{GHz}$ by changing the beam energy from 500 to 260 $\mathrm{kV}$. Interaction is only possible with the $\mathrm{TM}_{01}$ mode. Taking $\epsilon_{\mathrm{r}}=8.6$, the laser can be tuned from 44.9 to 49.5 $\mathrm{GHz}\left(\mathrm{TM}_{01}\right)$, from 151 to $162 \mathrm{GHz}\left(\mathrm{TM}_{02}\right)$ and from 262 to $275 \mathrm{GHz}\left(\mathrm{TM}_{03}\right)$ when the beam voltage decreases from 500 to $250 \mathrm{kV}$. Increasing the thickness of the dielectric liner results in a decrease of the synchronous frequency for a specific mode $(f \propto 1 / d)$.

\section{Non-linear simulation}

To estimate the performance of the Cherenkov FEL in the far infrared and millimeter wavelength region, nonlinear calculations were performed using a three dimensional model [5]. The parameters used in the calculations reflect the design parameters for the Cherenkov FEL under construction. They are shown in Table 1.

At two typical beam energies the maximum output power was calculated as a function of the frequency for a single pass amplifier configuration. The parameters used are shown together with the results in Fig. 2. For both beam energies only interaction with the lowest order $\mathrm{TM}_{01}$ is possible. At the first beam energy of $\gamma=1.80$, and using $d=0.5 \mathrm{~mm}$, a maximum output power of about $2.25 \mathrm{MW}$ at $138 \mathrm{GHz}$ was found, resulting in an cfficiency of $5.5 \%$. With an input power of $100 \mathrm{~W}$, this maximum power is reached with an interaction length of $33 \mathrm{~cm}$. The output power drops sharply towards zero for higher frequencies and much slower for decreasing frequency. The FWHM is
Table 1

Overview of the main parameters of the Cherenkov FEL under construction. $\tau$ is the electron pulse duration, $f_{\text {rep }}$ is the repetition frequency for the electron pulses, $l$ is the length of the dielectric medium and $d_{\mathrm{b}}$ is the thickness of the annular beam. Other symbols are explained in the text.

\begin{tabular}{lll}
\hline$V_{\mathrm{b}}$ & $\mathrm{kV}$ & $300-500$ \\
$r_{\mathrm{b}}$ & $\mathrm{mm}$ & $=3.0$ \\
$d_{\mathrm{h}}$ & $\mathrm{mm}$ & $<1$ \\
$I$ & $\mathrm{~A}$ & 100 \\
$\delta p_{z} / p_{z}$ & & $<1 \%$ \\
$\tau$ & $\mu \mathrm{s}$ & 10 \\
$f_{\text {rep }}$ & $\mathrm{Hz}$ & $\leq 10$ \\
$\epsilon_{\tau}$ & & $2.1-10$ \\
$r_{\mathrm{d}}$ & $\mathrm{mm}$ & 3.5 \\
$d$ & $\mathrm{~mm}$ & $0.25-0.5$ \\
$l$ & $\mathrm{~cm}$ & 30 \\
$\delta$ & $\mathrm{mm}$ & 0.5 \\
\hline
\end{tabular}

about $10 \mathrm{GHz}$ resulting in a linewidth of $\delta f / f=7.2 \%$. At the other beam energy of $\gamma=1.587$ a thickness of $d=0.25$ $\mathrm{mm}$ was used for the dielectric liner. Here a maximum output of $58 \mathrm{~kW}$ was obtained at a distance of about 69 $\mathrm{cm}$. The frequency is $356 \mathrm{GHz}$. Again the power drops rather sharply for higher frequencies and less quickly for lower frequencies. A FWHM of about $5 \mathrm{GHz}$ is found which corresponds to a relative linewidth of $\delta f / f=1.4 \%$. The maximum output power increases for lower frequencies, e.g. a maximum power of $7.1 \mathrm{MW}$ ( $20 \%$ efficiency) was found at $53.5 \mathrm{GHz}$ with the following parameters: $\gamma=1.685, \epsilon_{\mathrm{r}}=7.0$ and $d=0.5 \mathrm{~mm}$. To investigate the influence of the thickness $d_{\mathrm{b}}$ of the electron beam, the

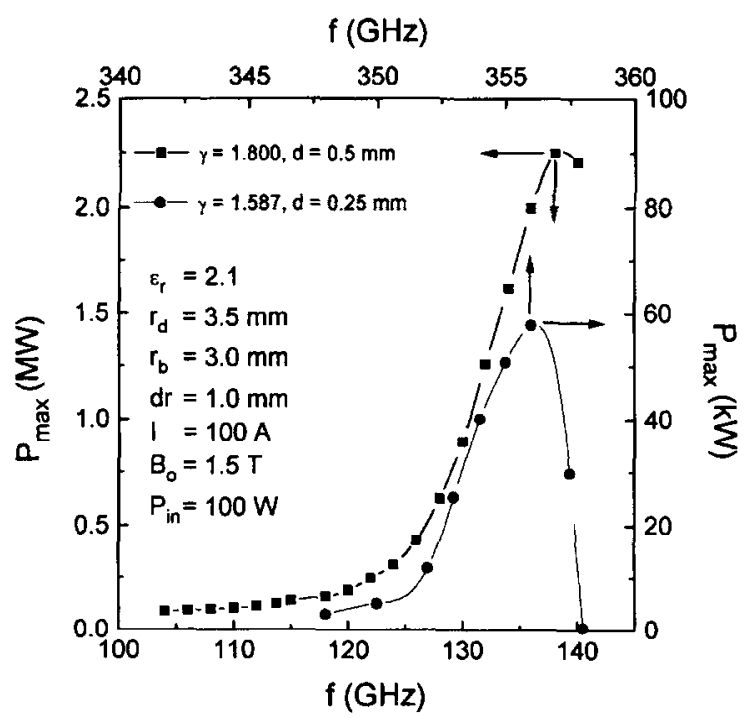

Fig. 2. Maximum power versus input frequency for two different beam energies. 
maximum output power was calculated as a function of $d_{\mathrm{d}}$. It was found that the maximum power dropped with a factor of 4 in going from an annular beam with $d_{\mathrm{b}}=0.5$ $\mathrm{mm}$ to a solid electron beam. The frequency used was 138 $\mathrm{GHz}$ (see Fig. 2). Reducing $d_{\mathrm{h}}$ to smaller values results in even higher powers than presented in Fig. 2. However, it becomes extremely difficult to produce high current, hollow electron beams of good quality with very small thickness.

Further calculations (not presented here) show that the laser is sensitive to the beam quality as found for other Cherenkov FELs [5]. Therefore a special electron gun was designed to produce a good quality, thin walled annular beam to be used in the Cherenkov FEL under construction.

\section{Thermionic electron gun}

Apart from the quality of the electron beam, the electron source must be reliable, reproducible, and operate at a maximum repetition rate of $10 \mathrm{~Hz}$. Therefore a thermionic gun powered by a modulator was designed for this project [6]. A lanthanum hexaboride thermoemitter was chosen in view of its good reproducibility, high repetition rate and well developed technology, insuring a long pulse with a current density up to $10 \mathrm{~A} / \mathrm{cm}^{2}$. The limited emissive power makes a rather large cathode surface necessary and a high degree of magnetic compression $(\approx 10)$ at a short inter-electrode distance.

A simplified electrode configuration with magnetic coils, the magnetic field produced by these coils and the corresponding beam behaviour are presented in Fig. 3 . The coil at the exit is necessary to test the gun separately and compensates for the solenoid which produces a constant magnetic field in the interaction region of the Cherenkov
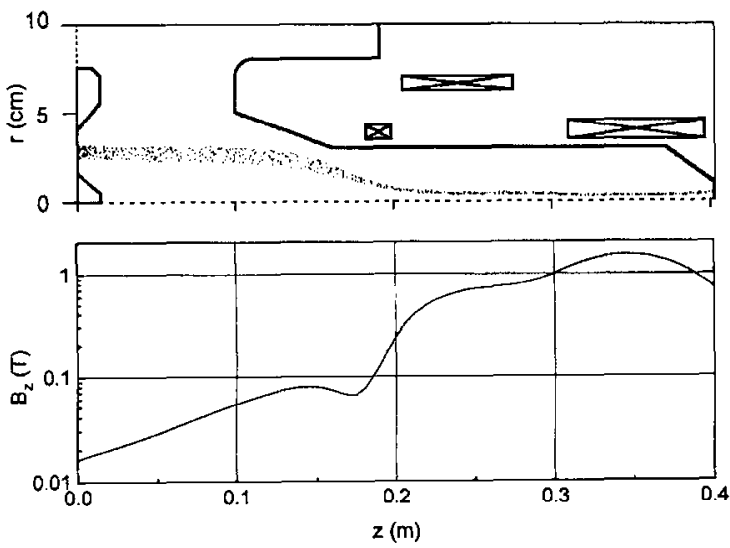

Fig. 3. Schematic layout of the electron gun showing the beam behaviour. Also shown is the magnetic field required for beam compression.
FEL. The first coil provides an opposite magnetic flux to create a correcting bump in the magnetic field profile. The low magnetic field at the cathode surface is not strong enough to link the electrons to the magnetic force lines. At the entrance of the anode hole (adiabatic region) they must be put on a particular force line to prevent transverse oscillations. The calculated microperveance was 0.525 $\mu \mathrm{A} / \mathrm{V}^{3 / 2}$.

The longitudinal momentum spread (see Table 1) was found to be rather sensitive with respect to coil position and currents. $\pm 1 \mathrm{~mm}$ displacement of one of the first two coils led to a significant increase in the spread and in the beam wall thickness. About the same effect was induced by $\pm 1 \%$ current variations in the coils. The effect of common displacements of the coils or common variations of the currents was weaker. The position of the third coil and current variations in it are not so crucial. An interesting feature of the chosen scheme is the scaling of coil exciting currents when changing accelerating voltage. For a fixed anode-cathode separation and variation of voltage from 200 to $500 \mathrm{kV}$, the beam structure remains practically unchanged if the coil currents are varied proportionally to the total momentum of particles corresponding to the applied voltage. This feature can turn out to be useful in crcating an overall control system.

Gun tests are currently carried out in a single-pulse regime on a special test facility based on a 10-stage open-air generator. The maximum test voltage of $300 \mathrm{kV}$ with a pulse flat top of $10 \mu \mathrm{s}$ duration is limited by the electric strength of the dividing transformer for the heater current. So far an emission current of over $100 \mathrm{~A}$ is reached for $1.8 \mathrm{~kW}$ consumed power $(20 \mathrm{~V}, 90 \mathrm{~A})$. In normal operation the gun will be powered by a modulator delivering voltage pulses up to $500 \mathrm{kV}$ at a maximum repetition rate of $10 \mathrm{~Hz}$. Low voltage tests of the modulator show a ripple of less than $0.5 \%$ during the flat part of the pulse. Preliminary results of the test confirm the main design considerations of the gun as far as they concern generation of a high current annular beam of a small diameter. However, peculiar problems are foreseen at a fine tuning stage because of an unprecedingly small longitudinal momentum spread to be measured and corrected.

\section{Acknowledgements}

The development of the electron gun has been supported by a grant from the Netherlands Organization for Scicntific Rescarch (NWO).

\section{References}

[1] K.J. Button (ed.), Infrared and Millimeter Waves, Vol. 13 (Academic Press, 1985). 
[2] J. Walsh, B. Johnson, G. Dattoli and A. Renieri, Phys. Rev. Lett. 53 (1984) 779.

[3] S. Jacobs (ed.), Physics of Quantum Electronics, Vol. 7 (Addison Wesley, 1980).

[4] E. Garate and J. Walsh. IEEE Trans. Plasma Phys. PS-13 (1985) 524
[5] H.P. Freund and A.K. Ganguly, Phys. Fluids B 2 (1990) 2506.

[6] A.V. Agafonov, E.G. Krastelev, A.N. Lebedev, V.S. Voronin, A.M. Bishaev, V.A. Krasnopolsky and P.J.M. van der Slot, 10th Int. Conf. on High Power Particle Beams, San Diego, CA, USA, 1994, to be published. 